\title{
DIRECCIÓN DE ARTE Y PRODUCCIÓN PUBLICITARIA: ANALIZAR, CONECTAR, EXPERIMENTAR Y CREAR
}

\section{Art direction and advertising production: analyze, connect, experiment and create}

Vanessa Roger-Monzó1: ESIC Business \& Marketing School. España. vanessa.roger@esic.edu

Fernando Castelló-Sirvent² ${ }^{2}$ ESIC Business \& Marketing School. España. fernando.castello@esic.edu

El presente trabajo ha sido desarrollado en el marco del grupo de investigación INDOESVAL, integrado en el Campus de Valencia de ESIC Business E Marketing School.

\section{RESUMEN}

En este trabajo se presenta una experiencia de innovación educativa basada en la creación de campañas de publicidad por parte de los alumnos sobre una temática previamente establecida por el docente. La experiencia se ha llevado a cabo en la asignatura 'Dirección de Arte y Producción Publicitaria', impartida en el $3^{\circ}$ curso del grado oficial en Comunicación y Relaciones Públicas durante el primer semestre del curso 2017-2018, en el campus de Valencia de ESIC Business \& Marketing School. El objetivo de este proyecto es fomentar el aprendizaje autónomo y continuo de los estudiantes, incrementar su participación activa y el trabajo colaborativo en el aula, así como vincularlos a las realidades profesionales de su entorno. El proceso se ha articulado a partir de una doble vertiente: el Modelo de Estilos de Aprendizaje de Felder-Silverman - FSLSM y el Modelo ACEC. Para ello, se ha empleado el cuestionario sobre el índice de Estilos de Aprendizaje (ILS) propuesto por Felder y Soloman. A partir de los resultados obtenidos se ha planteado una secuencia de acciones docentes basadas en cuatro ejes: analizar, conectar, experimentar y crear (ACEC), integrando las

\footnotetext{
1 Vanessa Roger-Monzó: Doctora en Comunicación Audiovisual por la Universidad Politécnica de Valencia. (2010). Licenciada en Ciencias de la Información -rama Imagen Visual y Auditiva- por el CEU San Pablo (2000), en Periodismo por la Universidad de Valencia (2005) y en Publicidad y Relaciones Públicas por la Universitat Oberta de Catalunya (2015).

${ }^{2}$ Fernando Castelló-Sirvent: Licenciado en Administración y Dirección de Empresas por la Universidad de Valencia (2003) y en Investigación y Técnicas de Mercado por la Universitat Oberta de Catalunya (2015). Graduado en Economía por la Universidad de Valencia (2017).
} 
Roger-Monzó V. y Castelló-Sirvent, F. Dirección de arte y producción publicitaria: analizar, conectar, experimentar y crear

preferencias del perfil cognitivo de los estudiantes según el FSLSM. El análisis cuantitativo de la experiencia revela una alta aceptación entre los alumnos.

PALABRAS CLAVE: experiencia de aprendizaje - innovación educativa - aprendizaje autónomo - perfil cognitivo - dirección de arte - producción publicitaria - EEES.

\begin{abstract}
This paper presents an educational innovation experience based on the creation of advertising campaigns by students on a subject previously established by the teacher. The experience has been carried out in the subject Art Direction and Advertising Production, given in the 3rd year of the official degree in Communication and Public Relations during the first semester of the 2017-2018 academic year, in the Valencia campus of ESIC Business \& Marketing School. The objective of this project is to promote the autonomous and continuous learning of students, increase their active participation and collaborative work in the classroom, as well as linking them to the professional realities of their environment. The process has been approached from two different perspectives: the Felder-Silverman Learning Styles Model FSLSM and the ACEC Model. For this, the questionnaire on the Learning Styles Index (ILS) proposed by Felder and Soloman has been used. Based on the results obtained, a sequence of teaching actions based on four axes has been proposed: analyze, connect, experiment and create (ACEC), integrating the preferences of the students' cognitive profile according to the FSLSM. The quantitative analysis of the experience reveals a high acceptance among the students.
\end{abstract}

KEY WORDS: learning experience - educational innovation - autonomous learning cognitive profile - art direction - advertising production - EHEA.

\title{
DIREÇÃO DE ARTE E PRODUÇÃO PUBLICITÁRIA: ANALISAR CONECTAR, EXPERIMENTAR E CRIAR
}

\section{RESUME}

Neste trabalho se apresenta uma experiência de inovação educativa baseada na criação de campanhas de publicidade por parte dos alunos sobre uma temática previamente estabelecida pelo docente. A experiência aconteceu na matéria Direção de Arte e Produção Publicitária, em partida no 3 curso do grau oficial em Comunicação e Relações Públicas durante o primeiro semestre do curso 2107/2018, no campus de Valência de ESIC Business \& Marketing School. O objetivo desse projeto é fomentar a aprendizagem autônoma e continua dos estudantes, incrementar sua participação ativa e o trabalho colaborativo na aula, assim como vincular -los às realidades profissionais do seu entorno. O processo foi articulado a partir de uma dupla vertente : O Modelo de Estilos de Aprendizagem de Felder -Silverman- FSLSM e o Modelo ACEC. Para isso, se

Vivat Academia. Revista de Comunicación. 15 junio 2019 /15 septiembre 2019, nº 147, 65-86 
Roger-Monzó V. y Castelló-Sirvent, F. Dirección de arte y producción publicitaria: analizar, conectar, experimentar y crear

amplou o questionário sobre o Índice de Estilos de Aprendizagem (ILS) proposto por Felder e Soloman. A partir dos resultados obtidos propuseram uma sequência de ações docentes baseadas em 4 eixos: analisar, conectar, experimentar e criar(ACEC), integrando as preferências do perfil cognitivo dos estudantes segundo o FSLSM. A análises quantitativa da experiência revela uma.alta aceitação entre os alunos.

PALAVRAS CHAVE: experiência de aprendizagem - inovação educativa, aprendizagem autônoma - perfil cognitive - direção de arte - produção publicitária EEES.

\section{Como citar el artículo:}

Roger-Monzó, V., y Castelló-Sirvent, F. (2019). Dirección de arte y producción publicitaria: analizar, conectar, experimentar y crear. [Art direction and advertising production: analyze, connect, experiment and create]. Vivat Academia. Revista de Comunicación, 147, 65-86. http://doi.org/10.15178/va.2019.147.65-86. Recuperado de http://www.vivatacademia.net/index.php/vivat/article/view/1126

\section{INTRODUCCIÓN}

La enseñanza universitaria debe idear experiencias innovadoras que fomenten la adquisición de nuevas habilidades, capacidades y aptitudes a través del autoaprendizaje. De este modo, además de transmitir conocimiento, el docente estimula el aprendizaje y brinda asesoramiento (Jiménez-Marín et al., 2012, p. 552). Así pues, las acciones docentes de las instituciones educativas superiores deben estar alineadas con el desarrollo de las destrezas que el alumnado desempeñará en su futura vida profesional.

En este sentido, el Espacio Europeo de Educación Superior (EEES) aborda la necesidad de establecer estrategias educativas orientadas a que el alumnado desarrolle un aprendizaje continuo y autónomo. De hecho, el Documento Marco sobre la Integración del Sistema Universitario Español en el EEES del Ministerio de Educación, Cultura y Deportes (2003, p. 7) señala que el ámbito universitario debe incluir la orientación profesional en la formación que aporta: "deberán proporcionar una formación universitaria en la que se integren armónicamente las competencias genéricas básicas, las competencias transversales relacionadas con la formación integral de las personas y las competencias más específicas que posibiliten una orientación profesional que permita a los titulados una integración en el mercado de trabajo".

Además, el aprendizaje debería "proponer formas amplias y estructuradas que permitieran la expansión personal y que también impliquen, a cada uno en particular, en la tarea de divertirse aprendiendo y en hacerlo de manera interesante y productiva para su desarrollo" (Botella y Adell, 2018, p. 112). En este sentido, las instituciones educativas deben centrar el concepto de conocimiento en el desarrollo de experiencias

Vivat Academia. Revista de Comunicación. 15 junio 2019 /15 septiembre 2019, nº 147, 65-86 
Roger-Monzó V. y Castelló-Sirvent, F. Dirección de arte y producción publicitaria: analizar, conectar, experimentar y crear

que faciliten el desempeño de procesos cognitivos vinculados a los proyectos vitales (Castillo y Marín, 2009). No en vano, diversos estudios revelan que el proceso de aprendizaje depende de aspectos personales, dado que todo individuo posee un estilo y características propias, que pueden transformarse con el tiempo y difieren según el contexto de las tareas educativas (Honey y Munford, 1986).

Así pues, los Estilos de Aprendizaje han sido definidos como "los rasgos cognitivos, afectivos y fisiológicos, que sirven como indicadores relativamente estables, de cómo los discentes perciben, interrelacionan y responden a sus ambientes de aprendizaje" (Alonso et al., 1994).

Por otro lado, existe un consenso en el ámbito docente sobre la necesidad de favorecer la participación activa y real del alumnado (Martínez de Miguel, 2007). Asimismo, se está otorgando gran importancia al trabajo cooperativo como metodología docente, lo que conlleva que el ámbito de la enseñanza universitaria se encuentre en proceso de transformación (Vallet et al., 2017). La introducción de los entornos virtuales de aprendizaje como Moodle favorece el trabajo cooperativo entre alumnos (GómezCamarero et al., 2010). Por tanto, y teniendo en cuenta la evolución en las necesidades formativas que demanda la sociedad, es indispensable promover experiencias innovadoras en los procesos de enseñanza-aprendizaje con el fin de capacitar al alumno en las destrezas que desempeñará durante su vida profesional, dado que el mercado laboral es muy cambiante y competitivo (Roger-Monzó et al., 2015).

\section{OBJETIVOS}

En este estudio se presenta una experiencia de innovación educativa basada en la creación de campañas de publicidad por parte de los alumnos sobre una temática previamente establecida por el docente. La experiencia se ha llevado a cabo en la asignatura 'Dirección de Arte y Producción Publicitaria', impartida en el $3^{\circ}$ curso del grado oficial en Comunicación y Relaciones Públicas durante el primer semestre del curso 2017-2018, en el campus de Valencia de ESIC Business \& Marketing School.

Los objetivos de este proyecto son: 1) fomentar el aprendizaje autónomo y continuo de los estudiantes; 2) incrementar su participación activa y el trabajo colaborativo en el aula; 3) vincularlos a las realidades profesionales de su entorno.

El proceso se ha estructurado a partir de una doble vertiente: en primer lugar, el Modelo de Estilos de Aprendizaje de Felder-Silverman - FSLSM (1988, 2002) como referencia fundamental; a partir de este modelo se ha elaborado una secuencia de acciones docentes basadas en el Modelo ACEC (Castelló-Sirvent y Roger-Monzó, 2017; 2018a; 2018b; Roger-Monzó y Castelló-Sirvent, 2018) articulado en torno a los ejes analizar, conectar, experimentar y crear, integrando las preferencias del perfil cognitivo

Vivat Academia. Revista de Comunicación. 15 junio 2019 /15 septiembre 2019, nº 147, 65-86 
Roger-Monzó V. y Castelló-Sirvent, F. Dirección de arte y producción publicitaria: analizar, conectar, experimentar y crear

de los estudiantes según el FSLSM. Para diagnosticar dichas preferencias se ha empleado el cuestionario sobre el índice de Estilos de Aprendizaje (ILS) propuesto por Felder y Soloman (1991).

El Modelo de Estilos de Aprendizaje FSLSM se ha convertido en un referente replicado por diversos investigadores. De este modo, se han realizado numerosos trabajos en los que el FSLSM ha sido aplicado a experiencias innovadoras relativas al elearning (Joseph y Abraham, 2017; Qodad et al., 2016; Muruganandam y Srinivasan, 2016; Truong, 2016; Fu y Li, 2014) y al contexto de enseñanza de idiomas (Jingyun y Takahiko, 2015).

Por su parte, el Modelo ACEC ha sido aplicado en el proceso de creación de argumentos y en el desarrollo de pensamiento lateral (Castelló-Sirvent y Roger-Monzó, 2017), en el diseño de políticas públicas (Castelló-Sirvent y Roger-Monzó, 2018a), para la mejora de empleabilidad de egresados de formación profesional en presencia del proceso de transformación digital e industria 4.0 (Roger-Monzó y Castelló-Sirvent, 2018), y a los efectos de diseño de acciones en el aula por parte de los alumnos (CastellóSirvent y Roger-Monzó, 2018b). El Modelo ACEC concede especial importancia al aprendizaje colaborativo y su enfoque secuencial pretende favorecer la integración profesional futura de los alumnos en el mercado laboral.

El sector de la comunicación y la publicidad está experimentando una profunda transformación que ha dado lugar a nuevas tendencias comunicativas que incide en los mensajes y usuarios, especialmente con la utilización sistemática de las redes sociales. De este modo, las acciones docentes propuestas buscan potenciar la reflexión en el alumnado para resolver problemas complejos que pueden encontrarse en el mercado laboral.

El proyecto se asentó en la creación de campañas de publicidad por parte del alumnado, a partir de las indicaciones docentes recibidas, e incluyó la realización de un spot, dos cuñas radiofónicas, tres propuestas gráficas para su publicación en prensa escrita, y es desarrollo de una estrategia de publicidad en redes sociales.

La premisa fundamental del trabajo pasó por fomentar el desarrollo de destrezas que pudieran vincularse a la realidad profesional del sector de la comunicación, por lo que la experiencia estuvo orientada a la interiorización por parte del alumnado del proceso de producción publicitaria, desde una forma eminentemente práctica.

\section{METODOLOGÍA}

Como ya se ha explicado, la experiencia docente desarrollada ha tenido como base el Modelo de Estilos de Aprendizaje de Felder-Silverman - FSLSM. Este modelo se

Vivat Academia. Revista de Comunicación. 15 junio 2019 /15 septiembre 2019, nº 147, 65-86 
Roger-Monzó V. y Castelló-Sirvent, F. Dirección de arte y producción publicitaria: analizar, conectar, experimentar y crear

organiza en 4 dimensiones y 2 estilos opuestos por cada dimensión: percepción (sensitivo-intuitiva), entrada (visual-verbal), procesamiento (activo-reflexiva) y comprensión (secuencial-global), hecho que permite clasificar a los alumnos en función de su habilidad para procesar, percibir, recibir, organizar y entender la información (Peña et al., 2002).

El diagnóstico de las preferencias FSLSM se ha realizado mediante el cuestionario sobre el Îndice de Estilos de Aprendizaje (ILS) propuesto por Felder y Soloman (1991). Con este instrumento es posible evaluar las preferencias de aprendizaje del alumnado en las cuatro dimensiones mencionadas anteriormente. En otras palabras, el test proporciona información de las fortalezas o dificultades que los encuestados pueden encontrar en el contexto académico. De los 21 alumnos encuestados se obtuvieron 18 test válidos.

Los estilos de aprendizaje identificados en el aula se presentan en su polarización y/o con preferencia por la izquierda (extremadamente polarizados o con preferencia moderada por estilos de aprendizaje de tipo activo, sensorial, visual o secuencial), así como indiferentes y polarizados o con preferencia por la derecha (con preferencia moderada o extremadamente polarizados por estilos de aprendizaje de tipo reflexivo, intuitivo, verbal o global).

Tabla 1. Hoja de perfil.

\begin{tabular}{l|c|c|c|c|c|c|c|c|c|c|c|c|c}
\hline \hline & 11 & 9 & 7 & 5 & 3 & 1 & 1 & 3 & 5 & 7 & 9 & 11 & \\
\hline Activo & & & & & & & & & & & & & Reflexivo \\
\hline Sensorial & & & & & & & & & & & & & Intuitivo \\
\hline Visual & & & & & & & & & & & & & Verbal \\
\hline Secuencial & & & & & & & & & & & & & Global \\
\hline \hline
\end{tabular}

Fuente: Felder y Soloman (1991).

De este modo, las puntuaciones ubicadas entre el 1-3 / 3-1 muestran un equilibrio apropiado para cualquiera de las dimensiones, mientras que las preferencias moderadas se sitúan en el intervalo 5-7. Los casos registrados con preferencias muy fuertes por un extremo se ubican en 9-11.

Los estilos de aprendizaje fueron definidos por Felder y Silverman $(1988,2002)$ y, de forma concisa, se concretan de la siguiente forma: 
Roger-Monzó V. y Castelló-Sirvent, F. Dirección de arte y producción publicitaria: analizar, conectar, experimentar y crear

El alumno activo antepone el trabajo en equipo y comprende mejor los conceptos aplicándolos, mientras que el alumno reflexivo prefiere trabajar sólo y pensar los conceptos, especialmente en primera instancia.

El alumno sensorial apuesta por el aprendizaje pautado, resolviendo problemas mediante métodos claramente establecidos y delimitados. En cambio, al alumno intuitivo le gustan los métodos innovadores que les permite descubrir posibilidades y relaciones entre conceptos.

El alumno visual recuerda y comprende mejor lo que ve, ya sean imágenes, contenidos audiovisuales o esquemas, mientras que el alumno verbal prefiere explicaciones escritas y habladas. Según Felder y Silverman $(1988,2002)$, la mayoría de las personas son aprendices visuales, si bien en las clases de las instituciones educativas superiores se presenta muy poca información visual, puesto que principalmente los alumnos escuchan clases magistrales y leen material escrito.

El alumno secuencial aprende a través de pasos lineales, es decir, en etapas o fases ordenadas lógicamente. El alumno global aprende a grandes saltos y establece relaciones entre distintas materias.

Como se ha comentado, el alumnado que muestra preferencias intermedias representa orientaciones neutras con respecto a los extremos expresados por cada par dicotómico. Por el contrario, el alumnado situado en preferencias polarizadas encuentra dificultades de atención y seguimiento de aquellas acciones docentes que se sustentan de forma predominante en el extremo contrario.

Llegados a este punto, respetando las premisas del Documento Marco del EEES que promueve una formación orientada a la futura integración del alumnado en el ámbito profesional y tomando en consideración el FSLSM, se optó por aplicar el Modelo ACEC, que se imbrica con las demandas del contexto educativo actual y se estructura en cuatro ejes: analizar, conectar, experimentar y crear.

Si no existen actividades docentes orientadas al extremo predominante en sus preferencias, estos alumnos pueden tener dificultades de aprendizaje (Ramírez y Rosas, 2014).

En la articulación del Modelo ACEC fue posible la atención específica del alumnado que mostró previamente una polarización o preferencia extrema en alguno de los Estilos de Aprendizaje planteados por Felder y Silverman (1988, 2002).

Vivat Academia. Revista de Comunicación. 15 junio 2019 /15 septiembre 2019, nº 147, 65-86 
Roger-Monzó V. y Castelló-Sirvent, F. Dirección de arte y producción publicitaria: analizar, conectar, experimentar y crear

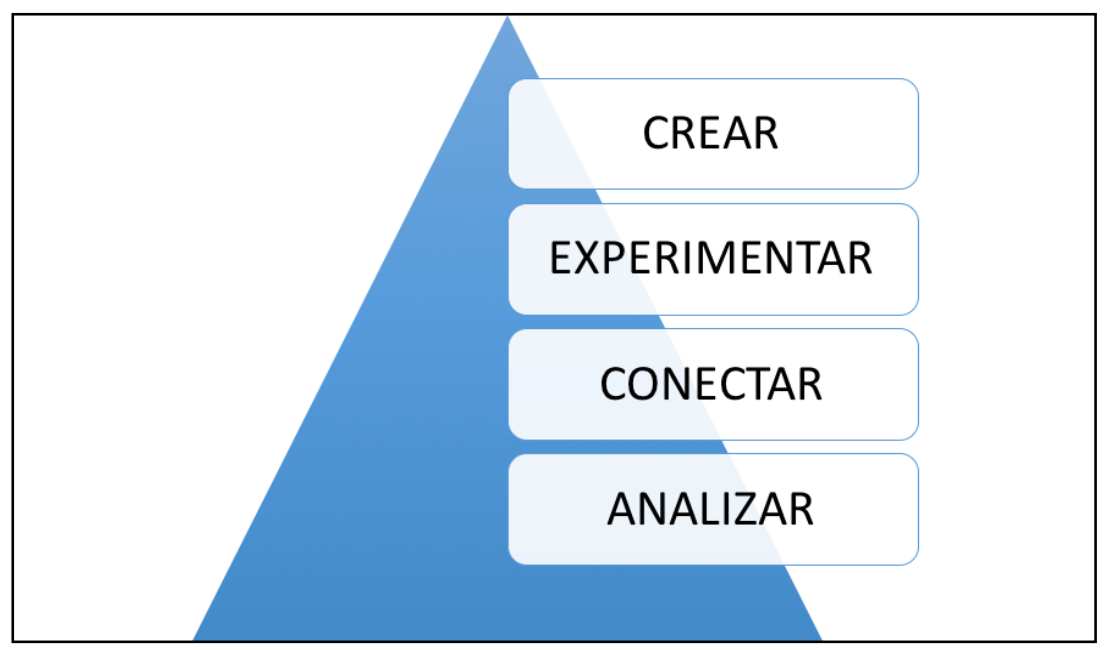

Gráfico 1. Etapas ACEC.

Fuente: elaboración propia.

A continuación, se describen los diferentes ejes ACEC.

\section{Etapa 1: ANALIZAR}

La articulación de acciones docentes de esta etapa tiene como finalidad el desarrollo en el alumnado de competencias transversales analíticas y prospectivas. La evolución de los conocimientos teóricos toma forma alrededor de este tipo soft skills relacionadas con el pensamiento crítico y la resolución de problemas (WEF y BCG, 2016). Así, las iniciativas se vuelven proactivas en torno a la formulación de un marco referencial futuro propio del área de conocimiento de los futuros egresados.

\section{Etapa 2: CONECTAR}

Los estudiantes abordan desafíos complejos propios de un contexto cambiante en tanto que conectan con la práctica profesional que se lleva a cabo en el presente en el área de especialización propia del campo de estudio en que se integra el currículo. Así, toman conciencia de iniciativas que se sitúan en la vanguardia profesional de innovación tecnológica $u$ organizativa en tanto que esta etapa integra cuatro de las competencias del siglo XXI definidas por el World Economic Forum en colaboración con Boston Consulting Group, en torno al conocimiento científico práctico, TIC y relacionadas con la conciencia social (WEF y BCG, 2016).

Las acciones docentes diseñadas e implementadas en esta etapa hacen posible que el alumno interiorice la dinámica competitiva del sector empresarial en el que se desempeñan las salidas profesionales de su disciplina en cuestión, propiciando en 
Roger-Monzó V. y Castelló-Sirvent, F. Dirección de arte y producción publicitaria: analizar, conectar, experimentar y crear

última instancia una observación reflexiva por parte del alumno que le permita identificar el presente y anticipar el futuro de su sector.

\section{Etapa 3: EXPERIMENTAR}

Las habilidades de carácter innovador y emprendedor de los alumnos son reforzadas por medio de acciones docentes diseñadas en esta etapa. Siguiendo una dinámica de adaptabilidad interdependiente al entorno, fomentan competencias de persistencia (WEF y BCG, 2016), al objeto de que los alumnos puedan llevar a cabo una completa experimentación en base a las herramientas y nuevas metodologías que se sitúan en la vanguardia profesional de su campo curricular.

Resulta central que en esta etapa el alumno pueda interactuar con el marco teórico previamente asimilado, proyectando en su comprensión las posibilidades competitivas disponibles.

Por medio de esta etapa el alumno alcanza la comprensión del proceso de generación de su propio conocimiento, ubicándole en el centro del proceso de aprendizaje como actor proactivo, no como receptor pasivo (Piscitelli et al., 2010). Resulta necesario alcanzar a través de un nivel de comprensión más profundo, los mecanismos que dan lugar a este proceso de aprendizaje basado en la experiencia (Nonaka y Takeuchi, 1995).

\section{Etapa 4: CREAR}

La imbricación del contenido curricular asimilado de forma previa, de base inicialmente teórica, así como la iteración de las tres fases precedentes, da lugar en esta etapa a la configuración de experiencias docentes que permitan la consolidación de competencias propias de rutinas innovadoras y de tipo disruptivo, integrando hasta cinco competencias propuestas por el World Economic Forum y Boston Consulting Group, en el despliegue de la creatividad que se deriva del estímulo continuado de la curiosidad del alumno, fomentando su propia iniciativa y estrategias de colaboración y desarrollo del liderazgo lateral (WEF y BCG, 2016). Esto es posible cuando los instrumentan esta fase como potente instrumento de creación de propuestas de valor que resulten plenamente competitivas en el mercado.

En esta etapa no se trata tanto de configurar experiencias docentes conducentes a fomentar la creatividad como de favorecer capacidades propias de rutinas innovadoras y de tipo disruptivo, haciendo posible que los alumnos consoliden

Por ello, el trabajo cooperativo con soporte TIC (Domingo y Fuentes, 2010, p. 172; González, 2016), resultan centrales para que los alumnos intensifiquen la experimentación de la filosofía "Do it yourself" (Piscitelli et al., 2010). 
Roger-Monzó V. y Castelló-Sirvent, F. Dirección de arte y producción publicitaria: analizar, conectar, experimentar y crear

\section{RESULTADOS}

\subsection{Resultados ILS}

En lo referido a los estilos de aprendizaje (ILS) detectados al aplicar el cuestionario desarrollado por Felder y Soloman el alumnado mostraba el siguiente perfil:

Tabla 2. Preferencias del alumnado encuestado según ILS.

\begin{tabular}{c|c|c|c|c|c|c}
\hline Dimensión/Perfil & $\mathbf{N}$ & $\begin{array}{c}\text { Preferencia } \\
\text { muy fuerte } \\
\text { por el } \\
\text { extremo } \\
\text { izquierdo }\end{array}$ & $\begin{array}{c}\text { Preferencia } \\
\text { moderada por } \\
\text { el extremo } \\
\text { izquierdo }\end{array}$ & $\begin{array}{c}\text { Equilibrio } \\
\text { apropiado } \\
\text { entre ambos } \\
\text { estilos de } \\
\text { aprendizaje }\end{array}$ & $\begin{array}{c}\text { Preferencia } \\
\text { moderada } \\
\text { por el } \\
\text { extremo } \\
\text { derecho }\end{array}$ & $\begin{array}{c}\text { Preferencia } \\
\text { muy fuerte } \\
\text { por el } \\
\text { extremo } \\
\text { derecho }\end{array}$ \\
\hline Activa/Reflexiva & 18 & 4 & 5 & 6 & 2 & 1 \\
\hline Sensorial/Intuitiva & 18 & 0 & 4 & 8 & 4 & 2 \\
\hline Visual/Verbal & 18 & 5 & 4 & 6 & 3 & 0 \\
\hline Secuencial/Global & 18 & 8 & 5 & 3 & 2 & 0 \\
\hline
\end{tabular}

Fuente: elaboración propia.

En la Tabla 2 se presenta el recuento de preferencias reveladas por el alumnado $(\mathrm{N}=18)$ tras la realización del test participante en la experiencia, siguiendo el cuestionario propuesto por Felder y Soloman (1991).

Se aprecia una marcada predominancia hacia perfiles activos, visuales y secuenciales, resultando el alumnado mayoritariamente equilibrado con respecto al binomio representado por el perfil sensorial-intuitivo.

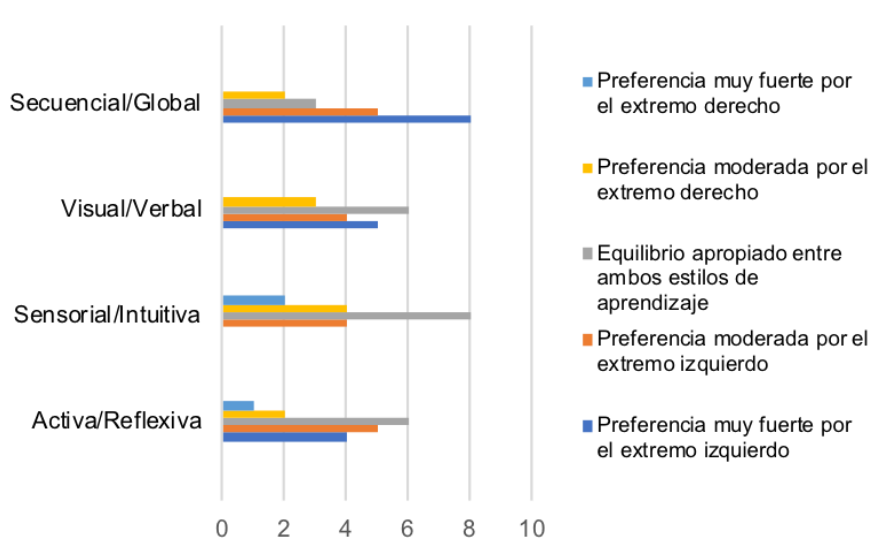

Gráfico 2. Preferencias del alumnado encuestado según ILS.

Fuente: elaboración propia.

Vivat Academia. Revista de Comunicación. 15 junio 2019 /15 septiembre 2019, n 147, 65-86 
Roger-Monzó V. y Castelló-Sirvent, F. Dirección de arte y producción publicitaria: analizar, conectar, experimentar y crear

Los diferentes cuadrantes recogen la suma de alumnos cuyas preferencias reveladas por los estilos de aprendizaje mostraban un equilibrio apropiado para cualquiera de las dimensiones con una puntuación ubicada entre 1-3 / 3-1, mientras que las preferencias moderadas situaban el recuento de sus valoraciones en el intervalo 5-7 correspondiente, y los casos registrados con preferencias muy fuertes por un extremo se situaban en el recuento de 9-11.

En atención a la diversidad existente en el aula, los docentes responsables de la experiencia llevaron a cabo la adaptación de las acciones docentes, a fin de vincular y facilitar el seguimiento por parte de todo el alumnado, teniendo especial interés en vincular a lo largo de todo el desarrollo de la experiencia con perfiles extremos, esto es, con preferencias muy fuertes en los extremos contrarios al diseño de la experiencia. Conocer los estilos de aprendizaje de los estudiantes facilita que el docente pueda articular los diferentes estilos de enseñanza disponibles.

La experiencia fue diseñada en base a las posiciones mayoritarias reveladas en el aula, resultando preponderante en el ámbito de perfiles de aprendizaje de tipo activo, intuitivo, visual y secuencial. Por este motivo, aquellos alumnos en preferencias moderadas y extremas para sus estilos antagónicos (reflexivo: 3; sensorial: 4; verbal: 3; global: 2) debieron ser involucrados de forma progresiva, diseñando acciones parciales cuyo objetivo pasaba por contar con su implicación.

\subsection{Acciones docentes integradoras}

Se optó por atender a los alumnos con polarización o preferencias extremas porque pueden tener dificultades de aprendizaje si no existen actividades docentes orientadas a su extremo (Ramírez y Rosas, 2014).

Cabe incidir sobre la relevancia que representa una vinculación efectiva y de participación activa por parte de todos los participantes en la experiencia, especialmente cuando el modelo de FSLSM sugiere que los perfiles extremos pueden no solo desconectar su atención, sino encontrar mayores dificultades para alcanzar los objetivos de aprendizaje planteados por una experiencia docente.

En este caso, al tratarse de un aula con un número muy reducido de alumnos, el peso porcentual de alumnos que se encontraban en posiciones extremas no era insignificante, sino que podía determinar el éxito global de la experiencia docente descrita. En particular, el porcentaje de alumnado que debió ser integrado oscilaba entre un $11 \%$ y un $22 \%$ (reflexivos: $16,67 \%$; sensoriales: $22,22 \%$; verbales: $16,67 \%$; globales: $11,11 \%$ ).

Estas acciones parciales de la experiencia docente se diseñaron en la implicación de recursos de aprendizaje con una preferencia reflexiva, sensorial, verbal y global. Las acciones específicas diseñadas a este fin también se basaron en el Modelo ACEC.

Vivat Academia. Revista de Comunicación. 15 junio 2019 /15 septiembre 2019, nº 147, 65-86 
Roger-Monzó V. y Castelló-Sirvent, F. Dirección de arte y producción publicitaria: analizar, conectar, experimentar y crear

\subsubsection{Acciones docentes basadas en el Modelo ACEC}

Las acciones docentes desarrolladas para integrar las preferencias mayoritarias del perfil cognitivo de los estudiantes activos, intuitivos, visuales y secuenciales en el marco del Modelo ACEC, se inició con la creación de equipos de trabajo para implementar una campaña publicitaria. Los grupos se mantuvieron a lo largo de todo el curso.

Las acciones en cada una de las etapas ACEC fueron las siguientes:

\section{Analizar:}

En esta etapa las acciones diseñadas tienen como finalidad que el alumno desarrolle habilidades prospectivas orientadas a vertebrar ulteriores iniciativas proactivas en su área de conocimiento.

- Como punto de partida, cada equipo realizó el análisis de un briefing de una marca real para, posteriormente, elaborar el correspondiente contrabriefing, con la finalidad de identificar los objetivos, audiencia y estrategia de comunicación de la campaña. (Campillo, 2012, p. 209).

\section{Conectar:}

Las experiencias docentes llevadas en esta etapa deben fomentar la reflexión y el pensamiento crítico en el alumno, para que sea capaz de identificar el presente y anticipar el futuro de su sector.

- A partir del trabajo anterior, cada grupo realizó una presentación visual en la que, a partir de la información deducida en el paso anterior, se explicaba el concepto y el eje de la campaña.

- Posteriormente, cada campaña era sometida a debate por parte de todos los alumnos con la finalidad de alcanzar las mejores opciones (Castelló-Sirvent y Roger-Monzó, 2017).

\section{Experimentar:}

En esta etapa los alumnos pudieron conocer y examinar nuevas metodologías y herramientas de vanguardia en su sector profesional. No en vano, resulta fundamental que la experiencia profesional penetre en el aula, dado que es la mejor forma de hacer sentir a los alumnos la esencia de su futuro ámbito laboral (Jiménez-Narros, 2012, p. 1254) y la metodología de enseñanza learning by doing centrada en la participación activa del estudiante es una opción óptima.

Por otro lado, todas estas acciones se diseñaron para que los alumnos las desarrollaran de forma colaborativa, dado que este aprendizaje proporciona mayor

Vivat Academia. Revista de Comunicación. 15 junio 2019 /15 septiembre 2019, n 147, 65-86 
Roger-Monzó V. y Castelló-Sirvent, F. Dirección de arte y producción publicitaria: analizar, conectar, experimentar y crear

rendimiento académico (Goikoetxea y Pascual, 2005). En este sentido, el uso de entornos virtuales como Moodle posibilita el trabajo cooperativo de los estudiantes (GómezCamarero et al., 2010).

- Elaboración de un spot:

- Puesto que uno de los aspectos a desarrollar en la campaña era la producción de un spot, la parte de preproducción del mismo implicó el desarrollo de un storyboard o guion gráfico para plasmar el discurso de manera visual y estimar su futura realización, teniendo en cuenta los preceptos marcados por el concepto y eje de la campaña. Para ello, el alumnado pudo experimentar con herramientas para confeccionar storyboards (StoryboardThat) y decidir la mejor alternativa para cada secuencia.

- A continuación, y en aras de realizar el rodaje del spot en un único día minimizando los imprevistos, cada equipo realizó un desglose de guion para conocer las necesidades en la grabación del spot. En esta parte del trabajo, el alumnado pudo comprobar la importancia de clasificar y contar con todos los recursos técnicos y humanos, así como con los elementos de atrezzo necesarios en el rodaje.

- Posteriormente, cada grupo llevó a cabo una investigación de las localizaciones en las que grabar. Para ello, tuvieron que desarrollar las correspondientes plantas de cámara que ayudaran a determinar con claridad la posición de los elementos del decorado, actores, cámara y demás accesorios de rodaje. Una vez acotadas las necesidades de la localización, los alumnos tuvieron que encontrar los escenarios más adecuados a sus necesidades y pedir los correspondientes permisos de rodaje, si así se requerían.

- Elaboración del plan de rodaje a partir de las necesidades detectadas en el desglose de guion y en el estudio de las localizaciones, estimando el tiempo necesario para la preparación de cada una de las secuencias y su posterior rodaje.

- Pruebas de edición en el aula de informática con un sistema de edición no lineal (Adobe Premiere) para experimentar las opciones de montaje con las que poder contar en la edición definitiva del spot.

- Elaboración de dos cuñas de radio: se ha considerado esencial integrar esta acción en la campaña publicitaria para acercar la industria radiofónica a los alumnos y brindarles "una visión real y crítica de la situación de la publicidad en el medio radiofónico con la intención de desarrollar sus aptitudes críticas, reflexivas y constructivas alrededor de la situación de la industria publicitaria radiofónica" (Rodero et al., 2011). En esta etapa, este proceso se ha llevado a cabo de la siguiente forma:

- Elaboración del guion técnico en base al concepto y eje de la campaña estipulado anteriormente. 
Roger-Monzó V. y Castelló-Sirvent, F. Dirección de arte y producción publicitaria: analizar, conectar, experimentar y crear

- Búsqueda de efectos sonoros y músicas en bancos de sonidos online para escoger los recursos más adecuados a su discurso.

- Realización de pruebas de grabación para determinar el tono y la voz más adecuada.

- Creación de tres gráficas para prensa:

- Los grupos trabajaron en el aula de forma colaborativa para determinar el diseño de las creatividades atendiendo al conjunto global de la campaña.

- Elaboración de una estrategia de redes sociales acorde con el contexto global de la campaña. Se ha incluido esta acción como parte de la campaña porque los alumnos de esta titulación deben "conocer las redes sociales como nuevo soporte publicitario e internet como uno de los fundamentales medios de comunicación en la creación de estrategias y, por lo tanto, el empleo de las mismas va más allá de los conocimientos que deberían tener los estudiantes de otro tipo de titulaciones" (Torres-Romay y Corbacho-Valencia, 2011, p. 789).

- Se hizo el análisis de redes sociales y de las páginas web de la propia marca y de la competencia a través de herramientas gratuitas para conocer su posicionamiento online y optar por las acciones de social media más adecuadas.

\section{Crear:}

Esta etapa es la más ambiciosa del Modelo ACEC, ya que las anteriores actividades docentes cristalizan en propuestas con gran valor académico y profesional: académico, porque los alumnos consolidan las competencias y habilidades desarrolladas en las fases precedentes; profesional, porque se presentan trabajos totalmente competitivos.

- Elaboración de un spot:

- Rodaje del spot en un día. El storyboard, desglose de guion, plan de rodaje y búsqueda de localizaciones anteriores facilitaron que esta fase se realizara en el menor tiempo posible, con la mayor calidad y profesionalidad, restando imprevistos.

- Visionado y edición del spot final en el aula de informática con un sistema de edición no lineal. El storyboard y las pruebas con Premiere realizadas anteriormente permitió que cada uno de los grupos optimizara el tiempo del que disponían para esta actividad y la llevaran a cabo con resultados de calidad profesional.

- Elaboración de dos cuñas de radio:

- Locución y grabación de las cuñas radiofónicas.

- Edición y sonorización de las cuñas radiofónicas. Como en el caso del spot, la búsqueda de recursos sonoros y las pruebas sonoras anteriores favoreció 
Roger-Monzó V. y Castelló-Sirvent, F. Dirección de arte y producción publicitaria: analizar, conectar, experimentar y crear

que esta acción se desarrollara de forma rápida. Se contaron con programas de edición de sonido para desarrollar dichas cuñas.

- Creación de tres gráficas para prensa:

- En el aula de informática, y de forma colaborativa, los alumnos ejecutaron las gráficas previamente diseñadas. Para ello, tuvieron a su disposición los softwares de retoque de imagen digital (Photoshop) y de diseño gráfico (Illustrator), cuyo uso había sido interiorizado en el curso anterior y durante el mismo semestre.

- Elaboración de una estrategia de redes sociales acorde con el contexto global de la campaña:

- Puesto que se trabajaban con marcas reales, no era factible materializar la estrategia en el canal online, por lo que cada grupo elaboró un dossier en el que se plasmaban las acciones de social media que habían estipulado en la campaña. El análisis anterior favoreció la propuesta de una estrategia de redes sociales factible y coherente con la campaña.

El momento culminante de esta experiencia se basó en la exposición de la campaña global con todas las piezas definitivas elaboradas y en una presentación corporativa. Esta estrategia de aprendizaje propia del ejercicio de la profesión publicitaria (JiménezMarín et al., 2012) permitió que cada grupo de trabajo contemplara todas las campañas de forma global.

De este modo, los alumnos pudieron comprender y comparar los conceptos y procesos de dirección de arte y producción publicitaria y acercar su trabajo a la realidad profesional.

\subsubsection{Modelo ACEC para perfiles minoritarios}

Como se ha señalado anteriormente, también se diseñaron acciones parciales de la experiencia docente con el fin de implicar el alumnado con una preferencia reflexiva, sensorial, verbal y global. Las acciones específicas diseñadas para dar respuesta a estos perfiles cognitivos a este fin también fueron diseñadas bajo el prisma del Modelo ACEC.

\section{Analizar:}

Para que los alumnos interiorizaran los aspectos teóricos de eje y concepto de una campaña, se recurrió al visionado y análisis de fragmentos de documentales de temática publicitaria y campañas de publicidad realizadas en diferentes soportes. A continuación, se realizaron puestas en común y debates dirigidos para que, de forma individual, los 
Roger-Monzó V. y Castelló-Sirvent, F. Dirección de arte y producción publicitaria: analizar, conectar, experimentar y crear

alumnos pudieran deducir conclusiones y establecer conexiones entre los diferentes aspectos de la dirección de arte y producción de una campaña publicitaria.

\section{Conectar:}

Los alumnos elaboraron ensayos individuales de carácter optativo para facilitar la reflexión sobre los factores esenciales de las campañas publicitarias analizadas en clase.

Se analizaron guiones técnicos de spots para, posteriormente, proponer distintas versiones de guiones para que, tras discutirlo en grupo, alumbrar el storyboard definitivo. Se siguieron los mismos procedimientos en la elaboración de los guiones de las cuñas radiofónicas, el diseño de gráficas y la propuesta de estrategia en redes sociales.

\section{Experimentar y crear:}

En estas etapas, las acciones docentes entre los distintos perfiles cognitivos fueron coincidentes con las descritas en el apartado anterior, ya que se diseñaron desde una perspectiva integral para ofrecer una cobertura global, aunque no superficial.

\subsection{Resultados del cuestionario de satisfacción de la experiencia docente}

El alumnado mostró valoraciones más elevadas en atención a cuestiones relacionadas con la calidad percibida por las prácticas ofrecidas por la experiencia docente $(4,39)$, seguida por la posibilidad de aprender nuevos contenidos $(4,17)$ y el estímulo intelectual representados por la experiencia $(4,11)$ y su duración deseada $(4,06)$, todas ellas con niveles de dispersión relativamente bajos (desviación típica entre 0,61 y 0,92).

Aun contando con valoraciones medias superiores a 3,5, las valoraciones menos elevadas en revelación de preferencias por parte del alumnado fueron: la capacidad para comprender el propio temario de las asignaturas $(3,5)$, la recomendación de la experiencia planteada $(3,56)$ y el deseo de realizar más experiencias docentes asociadas $(3,61)$.

En lo referido a esta última cuestión, la dispersión registrada se vuelve máxima (desviación típica de 1,04), junto a la cuestión referida a la integración del profesor en la experiencia docente (desviación típica de 1,20), hecho que indica una fuerte polarización dentro del aula, dando lugar a valoraciones mínimas (1 sobre 5) de un alumno para esta última cuestión, lo que provoca un sesgo importante en la horquilla de preferencias reveladas por el alumnado.

Vivat Academia. Revista de Comunicación. 15 junio 2019 /15 septiembre 2019, nº 147, 65-86 
Roger-Monzó V. y Castelló-Sirvent, F. Dirección de arte y producción publicitaria: analizar, conectar, experimentar y crear

Tabla 3. Resultados del cuestionario de satisfacción del proyecto.

\begin{tabular}{l|c|c|c|c}
\hline \multicolumn{1}{c|}{ Ítem } & Prom. & $\begin{array}{c}\text { Desv. } \\
\text { Típ. }\end{array}$ & Min. & Max. \\
\hline Me suscitaba interés la experiencia docente & 3,94 & 0,80 & 3 & 5 \\
\hline $\begin{array}{l}\text { Mi interés hacia los proyectos de innovación educativa } \\
\text { ha incrementado después de la experiencia docente }\end{array}$ & 3,89 & 0,83 & 3 & 5 \\
\hline $\begin{array}{l}\text { La estructura de la experiencia docente me ha parecido } \\
\text { correcta }\end{array}$ & 3,67 & 0,97 & 2 & 5 \\
\hline $\begin{array}{l}\text { La calidad de las prácticas adquiridas mediante la } \\
\text { experiencia docente }\end{array}$ & 4,39 & 0,61 & 3 & 5 \\
\hline $\begin{array}{l}\text { Se podría haber realizado una experiencia docente de } \\
\text { mayor duración }\end{array}$ & 4,06 & 0,80 & 3 & 5 \\
\hline $\begin{array}{l}\text { El profesor responsable nos ha integrado correctamente } \\
\text { en la dinámica de la experiencia docente }\end{array}$ & 3,83 & 1,20 & 1 & 5 \\
\hline $\begin{array}{l}\text { La experiencia docente me ha parecido } \\
\text { intelectualmente estimulante }\end{array}$ & 4,11 & 0,83 & 3 & 5 \\
\hline $\begin{array}{l}\text { La metodología de la experiencia docente me ha } \\
\text { permitido aprender contenidos que considero valiosos }\end{array}$ & 4,17 & 0,92 & 3 & 5 \\
\hline $\begin{array}{l}\text { Mue de otro modo no hubiese llegado a comprender } \\
\text { Mi interés por la materia estudiada en la asignatura ha } \\
\text { aumentado como consecuencia de la participación en la } \\
\text { experiencia docente }\end{array}$ & 3,78 & 1,00 & 2 & 5 \\
\hline $\begin{array}{l}\text { He comprendido mejor la teoría de comunicación } \\
\text { estudiada en el temario gracias a la experiencia docente }\end{array}$ & 3,50 & 0,86 & 2 & 5 \\
\hline $\begin{array}{l}\text { En conjunto, he mejorado la colaboración con otros } \\
\text { compañeros y con el profesor a través de la experiencia } \\
\text { docente }\end{array}$ & 3,89 & 0,90 & 3 & 5 \\
\hline $\begin{array}{l}\text { He mejorado mis competencias profesionales a lo } \\
\text { largo de la experiencia docente }\end{array}$ & 3,94 & 0,94 & 3 & 5 \\
\hline $\begin{array}{l}\text { Recomendaría una experiencia docente de estas } \\
\text { características a mis compañeros }\end{array}$ & 3,56 & 0,86 & 2 & 5 \\
\hline $\begin{array}{l}\text { Globalmente, estoy satisfecho de haber realizado esta } \\
\text { experiencia docente }\end{array}$ & 3,72 & 0,96 & 2 & 5 \\
\hline $\begin{array}{l}\text { Me gustaría que se realizaran más experiencias } \\
\text { docentes en otras asignaturas }\end{array}$ & 3,61 & 1,04 & 2 & 5 \\
\hline $\begin{array}{l}\text { Asistiría a una nueva experiencia docente que se } \\
\text { realizara en la universidad }\end{array}$ & 3,72 & 1,02 & 2 & 5 \\
\hline
\end{tabular}

Fuente: elaboración propia.

\section{DISCUSIÓN}

Tras desarrollar este trabajo se comprueba que es necesario ajustar las acciones docentes con respecto a los estilos de aprendizaje. Por un lado, el proyecto ha sido muy bien valorado por el alumnado participante, que ha incrementado sus niveles de participación y compromiso a lo largo del curso. Por ello, el diseño de experiencias 
Roger-Monzó V. y Castelló-Sirvent, F. Dirección de arte y producción publicitaria: analizar, conectar, experimentar y crear

docentes basadas en el FSLSM puede lograr una mayor y mejor vinculación del alumnado con su propio currículo, puesto que les ofrece la posibilidad de situarse en el centro de su aprendizaje, dado que se aspira a adaptarse a sus preferencias académicas.

Por otro lado, el diseño de las actividades docentes en base al Modelo ACEC favorece la adquisición de competencias relacionadas con el desarrollo profesional de su sector. Por otro lado, la experiencia actual y otras anteriores (Castelló-Sirvent y Roger-Monzó, 2017; Castelló-Sirvent y Roger-Monzó, 2018b), demuestran que existe un adecuado ajuste entre los estilos de aprendizaje FSLSM y ACEC, en tanto que modelo integrador.

No obstante, una limitación a tener en consideración de este trabajo viene representada por el tamaño de la muestra que, si bien recoge el universo poblacional, estadísticamente sugiere prudencia en la generalización de los resultados cuantitativos obtenidos. Se sugieren próximas investigaciones que aborden análisis cuantitativos con un mayor tamaño muestral que permita un contraste a partir de clústeres en base la caracterización sociodemográfica.

\section{REFERENCIAS}

Alonso, C., Gallego D. y Honey, P. (1994). Los Estilos de Aprendizaje: Procedimientos de diagnóstico y mejora. Bilbao: Ediciones Mensajero.

Botella Nicolás, A. Ma . y Adell Valero J. R. (2018). La integración de las artes a través de una propuesta didáctica en educación secundaria obligatoria: música, plástica y expresión corporal. Vivat Academia. Revista de Comunicación, 142, 109-123. doi: http://doi.org/10.15178/va.2018.142.109-123

Campillo Alhama, C. (2012). Aproximación al proceso didáctico de la Estrategia Publicitaria y de las Relaciones Públicas en el Espacio Europeo de Educación Superior. Estudios sobre el Mensaje Periodístico, vol. 18, $\mathrm{n}^{\mathrm{O}}$ especial octubre, 203-212. doi: http://dx.doi.org/10.5209/rev_ESMP.2012.v18.40926

Castelló-Sirvent, F., y Roger-Monzó, V. (2018a). ACEC: analizando, conectando, experimentando y creando políticas públicas. En XXXII Congreso Anual AEDEM. Gandia, 6-8 de junio.

Castelló-Sirvent, F., y Roger-Monzó, V. (2018b). El papel del alumnado en la propuesta y diseño de acciones docentes. @tic Revista d'Innovació Educativa, 20, 1-15.

Castelló-Sirvent, F., y Roger-Monzó, V. (2017). Construyendo argumentos: una experiencia innovadora para formar en competencias. En I Congreso Internacional de Formación del Profesorado e Innovación-Edutendencias. Madrid, 15-18 de noviembre.

Vivat Academia. Revista de Comunicación. 15 junio 2019 /15 septiembre 2019, nº 147, 65-86 
Roger-Monzó V. y Castelló-Sirvent, F. Dirección de arte y producción publicitaria: analizar, conectar, experimentar y crear

Castillo, I., y Marín, C. (2009). Hacia una visión holística de la educación universitaria: el amor y el caos un proceso creativo para el aprendizaje. Revista Electrónic@Educare, 13(1), 135-143.

Domingo, M., y Fuentes, M. (2010). “Innovación educativa: experimentar con las TIC y reflexionar sobre su uso" en Píxel-Bit. Revista de Medios y Educación, 36, 171-180.

Felder R. M., y Silverman, L. (1988, 2002). Learning and teaching styles in engineering education. Engineering Education, 78 (7), 674-681.

Felder, R. M., y Solomon, B. A. (1991). "Index of learning styles". Retrieved from www.ncsu.edu/effective_teaching/ILSdir/ILS-a.htm.

Fu, G. S. y Li, Y. F. (2014). Group differences: E-learning anxiety and sense of spatial orientation - Groups divided by Felder-Silverman Learning Style. Proceedings - 2014 International Conference of Educational Innovation Through Technology, EITT 2014,. 176183. doi: https://doi.org/10.1109/EITT.2014.36

Goikoetxea, E., y Pascual, G. (2005). Aprendizaje Cooperativo: Bases teóricas y hallazgos empíricos que explican su eficacia. Educación XX1, 5, 227-247. doi: https://doi.org/10.5944/educxx1.5.1.392

Gómez-Camarero, C., Palomares-Perraut, R., y Pino-Díaz, J. (2010). La utilización de herramientas colaborativas 2.0 en el ámbito de la documentación publicitaria. DIM: Didáctica, innovación y multimedia, 6(18).

González, M. (2016). “Formación docente en competencias tic para la mediación de aprendizajes en el proyecto canaima educativo//Teacher training in ICT skills in the mediation of learning in the Canaima Educational Project" en Telos, 18(3), 492-507. Recuperado de http://publicaciones.urbe.edu/index.php/telos/article/view/4691/5667

Honey, P., y Munford, A. (1986). Using your learning styles. Maidenhead: Peter Honey.

Jiménez-Marín, G., Elías-Zambrano, R., y Silva-Robles, C. (2012). Creatividad publicitaria y audiovisual a través de la web: proyectos de formación aplicada en el seno de la universidad. Estudios sobre el mensaje periodístico, vol. 18, n ${ }^{\mathrm{o}}$. especial noviembre, 551-558. doi: http://dx.doi.org/10.5209/rev_ESMP.2012.v18.40934

Jiménez-Narros, C. (2012). Actividades formativas de diseño gráfico orientadas a la adquisición de competencias profesionales: las terceras jornadas de periodismo 
Roger-Monzó V. y Castelló-Sirvent, F. Dirección de arte y producción publicitaria: analizar, conectar, experimentar y crear

audiovisual. Vivat Academia. Revista de Comunicación, 117E, 1252-1258. doi: http://dx.doi.org/10.15178/va.2011.117E.1252-1258

Jingyun, W., y Takahiko, M. (2015). The Reliability and Validity of Felder - Silverman Index of Learning Styles in Mandarin Version. Information Engineering Express, 1(3), 18.

Joseph L., y Abraham, S. (2017). Instructional design for learning path identification in an e-learning environment using Felder-Silverman Learning Styles Model. International Conference on Networks $\mathcal{E}$ Advances in Computational Technologies (NetACT), IEEE, pp. 215-220. doi: https://doi.org/10.1109/NETACT.2017.8076769

Martín, A., León, C., y García, A. (2014). Innovación docente para la integración de autoformación y autoevaluación en la plataforma web. Píxel-Bit. Revista de Medios y Educación, 44, 201-214. doi: http://dx.doi.org/10.12795/pixelbit.2014.i44.14

Martínez de Miguel, S. (2007). Una experiencia de innovación del portafolio del alumno en la diplomatura de educación social, desde el marco de la educación superior en Europa. Educatio Siglo XXI, 25, 125-144.

Ministerio de Educación, Cultura y Deportes (2003). La integración del Sistema Universitario Español en el Espacio Europeo de Enseñanza Superior. Documento Marco. Madrid: MECD.

Muruganandam S., y Srinivasan, N. (2016). Appraisal of Felder - Silverman Learning Style Model with Discrete Data Sets. Indian Journal of Science and Technology, vol. 9. doi: http://dx.doi.org/10.17485/ijst/2016/v9i10/88992

Nonaka, I., y Takeuchi, H. (1995). The knowledge-creating company: How Japanese companies create the dynamics of innovation. Oxford University Press.

Peña, C. I., Marzo, J. L., De la Rosa, J. L., y Fabregat, R. (2002). Un sistema de tutoría inteligente adaptativo considerando estilos de aprendizaje. Revista UIS Ingenierías, $1(2), 17-29$.

Piscitelli, A., Adaime, I., y Binder, I. (2010). El proyecto Facebook y la posuniversidad. Madrid: Ariel.

Qodad, A., Tetouan, E., Yadari, M. E. L., y Kenz, A. El. (2016). An Adaptive Learning System based on a Job Model, the Differentiated Instruction and Felder and Silverman's Learning Styles Model. Information Science and Technology (CiSt), 4th IEEE International Colloquium, 506-510.

Vivat Academia. Revista de Comunicación. 15 junio 2019 /15 septiembre 2019, nº 147, 65-86 
Roger-Monzó V. y Castelló-Sirvent, F. Dirección de arte y producción publicitaria: analizar, conectar, experimentar y crear

doi: http://dx.doi.org/10.1109/CIST.2016.7805100

Ramírez León, Y. del V., y Rosas Espín, D. (2014). Aplicación de la teoría de estilos de aprendizaje al diseño de contenidos didácticos en entornos virtuales. Etic@net, 2(14), 8.

Rodero, E., Larrea, O., y Vázquez, M. (2011). Integrando la formación de publicidad en radio en el contexto profesional. Vivat Academia. Revista de Comunicación, 117E, 14981516. doi: http://dx.doi.org/10.15178/va.2011.117E.1498-1516

Roger-Monzó, V., Guijarro-García, M., y Martí-Sánchez, M. (2015). “Digital Signage: An Experience of Innovation in Higher Education" en Multidisciplinary Journal for Education, Social and Technological Sciences, 2(2), pp. 1-13. doi: http://dx.doi.org/10.4995/muse.2015.3769

Roger-Monzó, V., y Castelló-Sirvent, F. (2018). Digital Transformation and Design of Teaching Activities for Cometency-based learning in superior grade formative courses. En INTED 2018 12th annual International Technology, Education and Development Conference. Valencia, 5-7 de marzo.

Torres-Romay, E., y Corbacho-Valencia, J.M. (2011). Redes sociales y docencia en los estudios de publicidad. Vivat Academia. Revista de Comunicación, 119, 71-82. doi: http://dx.doi.org/10.15178/va.2012.119.71-82

Truong, H. M. (2016). Integrating learning styles and adaptive e-learning system: Current developments, problems and opportunities. Computers in Human Behavior, 55, 1185-1193. doi: https://doi.org/10.1016/j.chb.2015.02.014

Vallet Bellmunt, T., Rivera Torres, P., Vallet Bellmunt, I., y Vallet Belmunt, A. (2017). Aprendizaje cooperativo, aprendizaje percibido y rendimiento académico de la enseñanza de marketing. Educación XX1, 20(1), pp. 277-297. doi: https://doi.org/10.5944/educxx1.1751

World Economic Forum y Boston Consulting Group. (2016). New Vision for Education: Fostering Social and Emotional Learning through Technology. Industry Agenda. World Economic Forum. Committed To Improving The State Of The World.

\section{AUTORES}

\section{Vanessa Roger-Monzó}

Doctora en Comunicación Audiovisual por la Universidad Politécnica de Valencia. (2010). Licenciada en Ciencias de la Información -rama Imagen Visual y Auditiva- por el 
Roger-Monzó V. y Castelló-Sirvent, F. Dirección de arte y producción publicitaria: analizar, conectar, experimentar y crear

CEU San Pablo (2000), en Periodismo por la Universidad de Valencia (2005) y en Publicidad y Relaciones Públicas por la Universitat Oberta de Catalunya (2015). Ha trabajado en diversos medios de comunicación en el ámbito de espacios informativos, programas y retransmisiones. Como investigadora, ha profundizado en la integración de nuevas tecnologías en la realización de contenidos audiovisuales, en el marketing online y en la didáctica de la comunicación, publicando artículos y participando en congresos nacionales e internacionales. Es profesora en el Departamento de Comunicación y Publicidad del campus de Valencia de ESIC Business \& Marketing School, donde forma parte de la Comisión de Titulación del grado en Comunicación y Relaciones Públicas y coordina la plataforma virtual Moodle del área de grado. vanessa.roger@esic.edu

Orcid ID: https://orcid.org/0000-0002-7498-0406

\section{Fernando Castelló-Sirvent}

Licenciado en Administración y Dirección de Empresas por la Universidad de Valencia (2003) y en Investigación y Técnicas de Mercado por la Universitat Oberta de Catalunya (2015). Graduado en Economía por la Universidad de Valencia (2017). Economista colegiado (COEV), está especializado en planificación estratégica y análisis económico, trabajando habitualmente como consultor y consejero de empresas, así como analista en el proceso de diseño de políticas públicas para la Administración. Como investigador, ha profundizado en temas de economía aplicada, dirección de empresas, emprendimiento e innovación docente. Profesor universitario en el Departamento de Economía y Finanzas del campus de Valencia de ESIC Business \& Marketing School. fernando.castello@esic.edu

Orcid ID: https://orcid.org/0000-0002-2088-0039 